\title{
Caractérisation des systèmes de production laitière et analyse des stratégies de valorisation du lait en milieu rural et périurbain au Niger : cas de la communauté urbaine de Niamey et de la commune rurale de Filingué
}

\author{
A.R. Boukary ${ }^{1}$ M. Chaïbou ${ }^{2}$ H. Marichatou ${ }^{2 *}$ G. Vias ${ }^{1}$
}

Mots-clés

Bovin laitier - Production laitière Produit laitier - Zone rurale - Zone périurbaine - Niger.

\begin{abstract}
Résumé
L'élevage et particulièrement la production du lait occupent une place prépondérante comme facteur de réduction de la pauvreté et de croissance économique. La présente étude a eu pour objectif de caractériser les systèmes de production et les processus de diversification vers le lait en milieu (péri) urbain [communauté urbaine de Niamey (CUN)] et en milieu rural [commune rurale de Filingué (CRF)] au Niger. Dans la CUN, des enquêtes ont été réalisées dans 35 sites laitiers choisis de manière aléatoire parmi les 150 déjà répertoriés sur un rayon de $50 \mathrm{~km}$ autour de la capitale. Un choix raisonné de 12 sites a permis d'administrer le questionnaire à 169 chefs de ménage. Dans la CRF, 49 chefs de ménage, répartis dans cinq villages, situés dans un rayon de $75 \mathrm{~km}$ autour de Filingué, ont été enquêtés. Les résultats ont montré que dans la CUN les éleveurs possédaient un petit nombre de vaches laitières (en moyenne cinq vaches, soit 28 p. 100 du cheptel bovin) ; la production s'étalait sur toutes les saisons et était de 7 à 10 l/ménage/jour ; le lait frais était plus souvent commercialisé qu'en CRF en raison de la présence d'unités de transformation laitière. En revanche, dans la CRF, les effectifs étaient plus importants (en moyenne 10 vaches laitières, soit 52 p. 100 du cheptel bovin); les femelles ne produisaient qu'en saison des pluies et en saison sèche froide (de 0 à $10 \mathrm{l} /$ ménage/jour pour 66 p. 100 des enquêtés, et entre 10 et 20 I pour 20 p. 100 des enquêtés) ; les produits laitiers étaient plus souvent transformés avant d'être vendus (beurre fondu, lait caillé, fromage). Les innovations observées chez les éleveurs enquêtés ont porté sur les transformations et les modifications de la conduite du troupeau. Les contraintes au développement de la production laitière relevaient, en milieu urbain, du problème de production et de conservation du lait frais de bonne qualité jusqu'au transformateur ou au consommateur et, en milieu rural, du problème de débouchés. Il est nécessaire, en milieu urbain, d'organiser l'approvisionnement en intrants alimentaires, la collecte du lait du soir et de procéder à une vulgarisation rapprochée de thèmes techniques et de pratiques innovantes.
\end{abstract}

1. ONG Karkara, Niamey, Niger.

2. Faculté d'Agronomie, université Abdou Moumouni, Niamey, Niger.

* Auteur pour la correspondance

Département productions animales, faculté d'Agronomie, BP 10960, université Abdou Moumouni, Niamey, Niger.

Tél. : +22793916531 / 20315237 ; fax : +227 20316612

E-mail : maricha@ refer.ne

\section{INTRODUCTION}

Dans plusieurs pays du globe et particulièrement en Afrique subsaharienne, les changements de l'environnement physique et climatique ainsi que l'explosion démographique ont entraîné une paupérisation très prononcée de la population avec pour corollaire une augmentation de la vulnérabilité et une migration massive des populations rurales vers les centres urbains. Très vite, la transformation des systèmes de production agricole est apparue comme 
une réponse à la détérioration du pouvoir d'achat des couches les plus défavorisées (9). Dans cette dynamique d'évolution des systèmes agraires, l'élevage et particulièrement la production du lait occupent une place prépondérante comme facteur de réduction de la pauvreté et de croissance économique. Au Niger, le lait est une composante stratégique pour des raisons historiques (le Niger est traditionnellement un pays d'élevage), nutritionnelles (source de protéines de bonne qualité) et économiques. En effet, le lait est une des rares spéculations qui permet quotidiennement une entrée d'argent, sans endommager le système qui le produit. La vente du lait et ses dérivés permet ainsi de maintenir l'animal en meilleur état et de procurer des revenus à tous les acteurs de la filière (producteurs, collecteurs, transformateurs et revendeurs). Si dans le contexte urbain nigérien quelques travaux assez récents (11) ont permis de mieux comprendre le fonctionnement de la filière lait, il n'en demeure pas moins que les investigations doivent être approfondies afin de mieux caractériser les acteurs et les processus de diversification aussi bien en milieu périurbain qu'en milieu rural, et d'étudier les interactions entre ces différents systèmes de production.

La présente étude a eu pour objectif la caractérisation des systèmes de production et des processus de diversification vers le lait en milieu périurbain [communauté urbaine de Niamey (CUN)] et en milieu rural [commune rurale de Filingué (CRF)] au Niger. Ce travail doit permettre d'identifier les contraintes et de proposer des options de développement.

\section{MATERIEL ET METHODES}

\section{Zones d'étude}

\section{Communauté urbaine de Niamey}

La CUN est localisée au bord du fleuve Niger dans la partie ouest du pays, entre $2^{\circ} 10^{\prime}$ et $2^{\circ} 14^{\prime}$ de long. E et $13^{\circ} 33^{\prime}$ et $13^{\circ} 36^{\prime}$ de lat. $\mathrm{N}$; elle couvre une superficie d'environ 12500 ha. Sur le plan administratif, Niamey est découpée en cinq communes et intègre les villages situés dans sa périphérie proche. La pluviométrie moyenne est de $545 \mathrm{~mm}$ et la saison des pluies dure trois à quatre mois (juin à septembre). Les températures annuelles sont très élevées : la température maximale moyenne se situe à $36,02{ }^{\circ} \mathrm{C}$, la minimale moyenne est de $22,13{ }^{\circ} \mathrm{C}$. Le réseau hydrographique de la CUN est marqué principalement par le fleuve Niger qui traverse la ville sur environ $15 \mathrm{~km}$ et auquel s'ajoutent quelques mares permanentes et de nombreuses mares temporaires.

Sur le plan morphopédologique, la CUN est constituée des plateaux cuirassés entaillés par la vallée du fleuve avec des bas-fonds temporairement gorgés d'eau et présentant des sols hydromorphes, des terrains sableux ou latéritiques par endroit et des sols ferrugineux tropicaux couvrant l'essentiel de la zone agricole.

On y rencontre en général une végétation arbustive clairsemée et des herbacées à apparition saisonnière. Dans les bas-fonds, la nature du sol et la proximité de l'eau sont propices au développement d'une végétation naturelle assez dense.

L'agriculture est pratiquée jusqu'à présent de manière traditionnelle et occupe une bonne frange de la population. Les principales cultures conduites en irrigation sont : la riziculture (630 ha), le maraîchage (400 ha) et l'arboriculture (450 ha). L'élevage occupe une place prépondérante dans l'activité de la population. Le cheptel a été estimé en 2004 à environ 25204 bovins, 72770 ovins, 44600 caprins, 330 camelins, 1700 asins et 3600 équins, sans compter un nombre important de volailles (7).

Sur le plan démographique, Niamey a connu ces dernières décennies, à l'instar des grandes villes africaines, une croissance et une urbanisation rapides. Cette ville, qui compte actuellement près d'un million d'habitants, devrait atteindre 2 à 2,5 millions en 2025 (3).

\section{Commune rurale de Filingué}

La CRF est située à $175 \mathrm{~km}$ de la capitale, toujours dans la zone agropastorale. Le secteur primaire occupe l'essentiel de la population composée d'agriculteurs, d'agropasteurs et de nomades. L'exode y est cependant très développé. La CRF fait partie du département de Filingué situé dans la partie nord-est de la région de Tillbéry, entre les parallèles $13^{\circ} 40^{\prime}$ et les méridiens $2^{\circ} 45^{\prime}$ et $4^{\circ} 20^{\prime}$. Il s'étend sur une superficie de $26813 \mathrm{~km}^{2}$. Les précipitations, assez variables dans le temps et dans l'espace, débutent véritablement dans la dernière décade de juin et durent trois à quatre mois. De 1984 à 1999, la pluviométrie moyenne à Filingué a été de $292,6 \mathrm{~mm}$ en 26 jours. La température maximale moyenne est de $41{ }^{\circ} \mathrm{C}$ en avril-mai et la moyenne minimale, observée en octobre-novembre, est de $38^{\circ} \mathrm{C}$. Filingué est situé en grande partie sur des plateaux constitués de grès argileux, de dunes stabilisées et indurées dans la partie occidentale, et de dunes vives dans la partie orientale. Ce complexe est traversé en son centre par une vallée sèche, le Dallol Bosso.

L'agriculture est la première activité économique du département et est pratiquée par 97 p. 100 de la population estimée à 405918 habitants en 2000. Les principales cultures sont le mil, le sorgho, le niébé, le gombo et l'oseille. L'élevage est la deuxième activité dans le département. Le cheptel est composé de l'ensemble des espèces traditionnelles élevées au Niger, notamment les bovins, les ovins, les caprins, les camélins, les équins, les asins. L'essentiel des aires de pâturage se situe dans la zone Nord pastorale ; dans la zone agropastorale, elles se limitent aux jachères, aux plateaux impropres à l'agriculture et à quelques rares espaces incultes (7).

\section{Collecte des données}

Les données ont été collectées au moyen d'enquêtes informelles et formelles. L'approche systémique a été privilégiée tout au long du processus, en considérant la filière lait comme partie intégrante du système agraire. Ainsi, pour décrire la diversité des systèmes de production qui caractérise l'élevage laitier, trois pôles caractéristiques ont été considérés : le ménage (l'éleveur ou le groupe social selon le niveau d'analyse), le troupeau et le territoire pastoral. Il s'agissait de décrire les interactions possibles existant entre ces pôles, mais aussi d'étudier les pratiques de valorisation du lait, mises en œuvre par les éleveurs. Des enquêtes ont été réalisées dans le but de confirmer et de compléter la compréhension des systèmes d'élevage laitier acquis, suite à une analyse documentaire préalablement établie.

\section{Enquêtes informelles au niveau de la CUN}

Cent cinquante sites regroupant 1562 unités de production laitière, répertoriées par Cantafora (2) sur un rayon de $50 \mathrm{~km}$ autour de Niamey, ont servi à constituer la base de sondage. Réalisée en assemblée, l'enquête a porté sur 35 de ces sites choisis par un échantillonnage aléatoire simple. A cet effet, les points clés de l'entretien ont été consignés dans un guide flexible portant sur : les principales activités agricoles et non agricoles (importance relative, occupation du temps, calendrier saisonnier), les pratiques de production actuelles et les raisons de leur adoption (en cas de changement observé), l'influence des facteurs endogènes et exogènes sur les systèmes de production, les obstacles rencontrés par les éleveurs, leur attitude face au risque et les méthodes utilisées pour résoudre les problèmes, et l'effet de chaque facteur sur les objectifs individuels en matière de production, de revenus et d'application de nouvelles techniques (8). Après chaque entretien, les renseignements récoltés ont été notés sur une fiche récapitulative. 
Ces données ont été par la suite codifiées et saisies sur le logiciel Excel en vue d'un traitement statistique ultérieur.

\section{Enquêtes informelles au niveau de la commune rurale de Filingué}

La démarche suivie a été la même que celle adoptée à la CUN, mais, en plus des producteurs, des entretiens ont eu lieu avec les services techniques de la région et les chefs coutumiers sur des questions se rapportant à l'origine des peuplements, les découpages administratifs et leur évolution dans le temps.

\section{Enquêtes formelles}

A la suite du traitement des données de l'enquête informelle, 12 sites laitiers ont été choisis pour le bassin laitier de Niamey. Un entretien structuré, basé sur un questionnaire approfondi, a été administré à 169 chefs de ménage. Les informations recherchées auprès du groupe cible ont pu être réparties en six catégories comme suit : caractérisation des éleveurs, composition et structure du troupeau, conduite de l'élevage, production du lait, stratégies de valorisation et commercialisation du lait, contraintes majeures rencontrées et projets d'amélioration.

Pour la commune rurale de Filingué, la même approche méthodologique a été adoptée et un questionnaire spécifique et adapté au contexte a été élaboré à cet effet. Mais, dans l'ensemble, les informations recherchées reprenaient les catégories précédentes. Au total 49 chefs de ménage, repartis dans cinq villages, situés sur un rayon de 75 km de Filingué, ont été interviewés.

\section{Analyse statistique}

Toutes les variables ont été préalablement codifiées et les données ont été d'abord saisies à l'aide du tableur Excel puis transférées sur les tableurs Minitab et Stata pour les besoins de traitements statistiques. Sur le logiciel Minitab, des analyses descriptives et de corrélation ont été effectuées entre les différentes variables afin d'obtenir le choix des variables à introduire dans le traitement statistique. Une catégorisation des sites laitiers a été faite à partir des critères liés à la conduite des troupeaux laitiers et des stratégies des éleveurs. Des tabulations et des comparaisons de moyennes (test de Student) ont été effectuées avec Stata.

\section{RESULTATS}

\section{Typologie des systèmes de production laitière en milieu périurbain}

Au sein de la CUN et de sa périphérie, les caractéristiques des sites laitiers (pratiques d'alimentation, stratégie de commercialisation, soins des animaux, répartition du travail au sein des ménages, degré d'intensification...) ont fait ressortir deux types de classifications : l'une correspondant aux sept grands axes routiers reliant Niamey aux autres sites urbains nationaux et internationaux, et l'autre correspondant aux trois couronnes ou strates établies en fonction de la distance qui sépare le site de la ville, chacune définissant un système d'élevage particulier (figure 1).

Couronne 1 : élevages de type «moderne » intra-urbains (Kirkissoye, coopérative laitière, particuliers...) appartenant à des commerçants, des fonctionnaires, des retraités et possédant souvent de gros moyens. C'étaient les sites d'Aéroport, de Banifandou, Saga, Pays bas...

Couronne 2 : ceinture de sites situés à la périphérie proche de la ville $(0$ à $9 \mathrm{~km})$. Ces sites avaient pour caractéristique principale leur implantation sur des terrains privés appartenant à la commune (espaces verts, terrains en voie de lotissement, parcelles privées). Les populations s'étaient en général implantées à une date récente.
Les troupeaux étaient divisés en deux groupes et seules les vaches en production étaient gardées sur place, le reste allant en transhumance sous la garde d'un ou de plusieurs membres de la famille. C'étaient les sites de Banifandou II, Talladjé, Kouara-Tégui, Route Filingué, Dar-es-salam...

Couronne 3 : sites situés entre 10 et $30 \mathrm{~km}$ de Niamey. C'étaient des villages où les populations étaient installées depuis longtemps. L'agrandissement de plus en plus important de la ville de Niamey était la cause de leur rapprochement et certains étaient en phase de devenir des quartiers de la capitale. C'étaient les sites de Jajiré, Feto-Bokki, Tokabinkané, Gorou Kirey...

Par rapport au nombre d'exploitants laitiers enquêtés, la couronne 3 était la plus peuplée car elle concentrait à elle seule presque la moitié des éleveurs interviewés, soit 46,7 p. 100 d'entre eux. Venait ensuite la couronne 2 qui comptait 32 p. 100 de l'effectif total. Ces deux couronnes montraient des situations globales de ces élevages laitiers traditionnels qui se trouvaient en zones urbaine et périurbaine de la CUN. La couronne 1 (exploitations laitières intra-urbaines) a concerné 21,3 p. 100 des enquêtés.

Les sites visités étaient regroupés autour de sept axes principaux. Chaque axe représentait un réseau routier qui reliait la ville de Niamey à une autre ville et/ou un village (axes de sortie de Niamey).

\section{Caractéristiques des élevages laitiers}

Les Peuls représentaient l'ethnie majoritaire parmi les éleveurs laitiers de la CUN et de sa périphérie, suivis des Zarmas et des Kourtés (5 p. 100), tandis que dans la CRF c'étaient les Haoussas et les Peuls qui dominaient. Dans la CUN, seules les deux ethnies majoritaires se retrouvaient dans les trois couronnes (tableau I).

Globalement, les exploitants enquêtés étaient très faiblement instruits : plus de 50 p. 100 en moyenne étaient analphabètes, et respectivement 6,5 et 0 p. 100 avaient fréquenté l'école (tous niveaux confondus) dans la CUN et la CRF. Cependant, la proportion des répondants arabisés était assez importante dans la CUN,

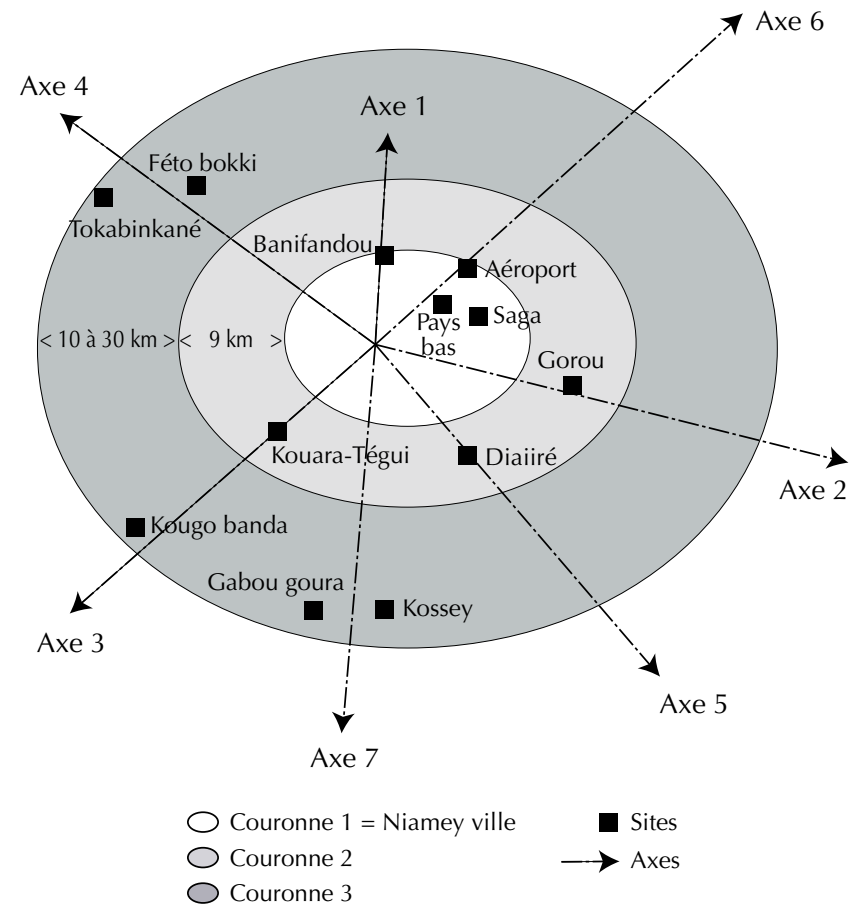

Figure 1 : typologie des systèmes d'élevage. 


\section{Tableau I}

Composition ethnique et niveau d'instruction des éleveurs

\begin{tabular}{lcc} 
Ethnie & Niamey (\%) & Filingué (\%) \\
\hline Haoussa & 0,59 & 47,00 \\
Kourté & 5,33 & $/$ \\
Mossi & 0,59 & $/$ \\
Peulh & 84,02 & 41,00 \\
Touareg & 0 & 4,00 \\
Zarma & 9,47 & 8 \\
Total & 100 & 100 \\
& & \\
Niveau d'éducation & & \\
Aucun & 50,30 & 76,00 \\
Alphabétisé & 12,43 & 10,00 \\
Primaire & 2,96 & 0 \\
Secondaire & 1,78 & 0 \\
Supérieur & 1,78 & 14,00 \\
Arabisé & 30,77 & 100 \\
Total & 100 &
\end{tabular}

puisqu'elle était environ de 31 p. 100 contre 14 p. 100 dans la CRF Cette dernière modalité d'instruction s'est révélée être dominante en matière d'éducation des enfants, surtout chez les Peuls.

Les principales activités pratiquées par la population étaient l'élevage laitier (100 p. 100 des ménages), l'agriculture ( 88 p. 100) et le maraîchage (11 p. 100). Les éleveurs de la CUN étaient très souvent structurés et possédaient des associations locales (49,3 p. 100), 40 p. 100 n'étaient pas organisés et 10,7 p. 100 étaient dans des groupements d'intérêt économique. Les Peuls, essentiellement, pour des raisons socio-économiques et/ou des mesures de sécurité, s'organisaient en association au sein de leur groupement pour renforcer, d'une part, le lien de fraternité et, d'autre part, limiter les éventuels conflits comme ceux entre agriculteurs et éleveurs.

\section{Caractéristiques du cheptel laitier}

\section{Modes d'acquisition du cheptel}

Ils étaient identiques aussi bien en milieu rural qu'en milieu périurbain, avec les mêmes profils en termes d'importance relative (figure 2). Le mode d'acquisition du troupeau laitier variait surtout en fonction des ethnies présentes. L'achat des animaux (18 et 24 p. 100 pour la $\mathrm{CRF}$ et la $\mathrm{CUN}$ ) était une pratique rencontrée dans tous les sites visités et était presque le seul mode chez les Haoussas et les Zarmas. Chez les Peuls, la formation du bétail était surtout fondée sur l'héritage familial (50 p. 100 et 39 p. 100, respectivement pour la CRF et la CUN). Par ailleurs, toujours chez cette ethnie, le habbanayé était aussi très fréquent (19 et 27 p. 100 pour la CRF et la CUN). Il consistait, pour un propriétaire d'animaux, à confier à un membre de sa famille une génisse ; ce dernier la lui rendait après la mise bas d'une velle qui lui revenait de droit (figure 2).

\section{Composition et taille du troupeau}

Les races bovines zébus étaient rencontrées dans les élevages laitiers, notamment les races Azawak (33 p. 100), Djelli (43 p. 100), Bororo (10 p. 100) et Goudali (3 p. 100). Il y avait aussi des races métisses issues de croisements entre races existantes (11 p. 100). En milieu rural, les troupeaux étaient assez souvent mixtes avec des effectifs spécifiques (tableau II).

Le tableau III montre que les éleveurs possédaient dans la CUN un petit nombre de laitières (en moyenne cinq vaches, soit 28 p. 100 du cheptel bovin), alors qu'en milieu rural, les effectifs étaient plus importants (en moyenne 10 vaches laitières, soit 52 p. 100 du cheptel bovin). Par ailleurs, au niveau de la CUN les éleveurs produisaient en toute saison (7 à 10 1/ménage/jour en moyenne) (tableau IV) et commercialisaient davantage le lait frais du fait de la présence d'unités de transformation laitière, alors

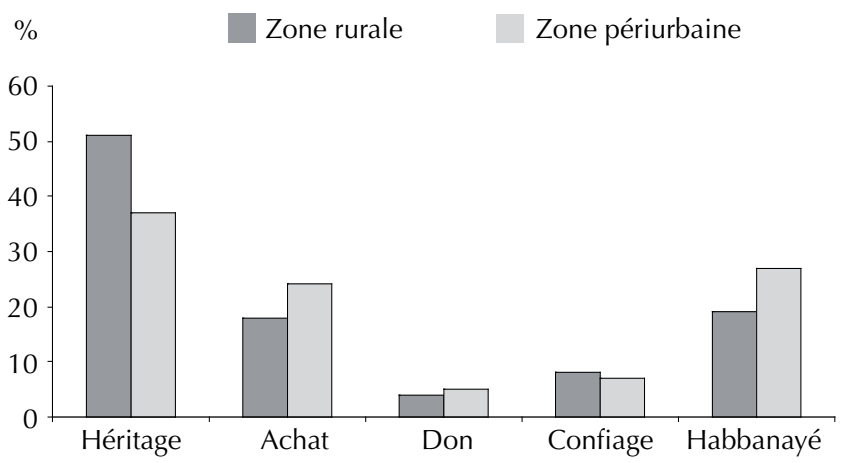

Figure 2 : modes d'acquisition du cheptel.

Tableau II

Taille moyenne du troupeau par espèce

$\begin{array}{lcc} & \text { Moyenne } & \text { Ecart-type * } \\ \text { Bovins } & 19 & 22 \\ \text { Caprins } & 21 & 25 \\ \text { Ovins } & 17 & 22 \\ \text { Camélins } & 0,6 & 1,25 \\ \text { Anes } & 2 & 2 \\ \text { Chevaux } & 0,32 & 0,98\end{array}$

* Les écart-types assez importants montrent la grande variabilité des effectifs en milieu rural

\section{Tableau III}

Proportion d'éleveurs en fonction des effectifs de femelles laitières

\begin{tabular}{lcc} 
Effectifs & \multicolumn{2}{c}{ Proportion des ménages } \\
\hline & CRF (\%) & CUN (\%) \\
\hline à 5 & 43 & 87,80 \\
6 à 10 & 30 & 11,90 \\
11 à 20 & 17 & 0,30 \\
20 à 40 & 6 & $/$ \\
$>41$ & 4 & $/$ \\
Total & 100 & 100
\end{tabular}

CRF : commune rurale de Filingué ; CUN : communauté urbaine de Niamey 
Tableau IV

Lait produit par ménage

\begin{tabular}{|c|c|c|c|c|}
\hline \multirow{3}{*}{$\begin{array}{l}\text { Quantité } \\
\text { de lait/ } \\
\text { ménage/ } \\
\text { jour (I) }\end{array}$} & \multicolumn{4}{|c|}{ Fréquence d'éleveurs concernés (\%) } \\
\hline & \multicolumn{3}{|c|}{ CUN } & CRF \\
\hline & $\begin{array}{c}\text { Saison } \\
\text { des pluies }\end{array}$ & $\begin{array}{l}\text { Saison } \\
\text { sèche froide }\end{array}$ & $\begin{array}{l}\text { Saison } \\
\text { sèche chaude }\end{array}$ & \\
\hline 0 à 5 & 31,36 & 30,18 & 48,53 & 29 \\
\hline 5,5 à 10 & 35,50 & 43,20 & 40,83 & 37 \\
\hline 10,5 à 20 & 27,81 & 20,70 & 10,18 & 20 \\
\hline 20,5 à 40 & 5,33 & 5,92 & 0 & 10 \\
\hline$>40$ & 0 & 0 & 0 & 4 \\
\hline Total & 100 & 100 & 100 & 100 \\
\hline
\end{tabular}

CUN : communauté urbaine de Niamey ; CRF : commune rurale de Filingué

qu'en milieu rural les femelles ne produisaient qu'en saison des pluies (pour 98 p. 100 des enquêtés) et en saison sèche froide (pour 97 p. 100 des enquêtés) (de 0 à 10 1/ménage/jour pour 66 p. 100 des enquêtés, et entre 10 et 20 1/ménage/jour pour 20 p. 100 d'entre eux), et les produits laitiers étaient plus souvent transformés avant d'être vendus (beurre fondu, lait caillé, fromage).

Pour la couronne 1 de la CUN une moyenne de $10 \pm 21$ par producteur a été enregistrée suivant les saisons, alors que pour les couronnes 2 et 3 cette moyenne était proche de $7 \pm 21$; le volume de la production laitière par exploitant était proportionnel à l'effectif des vaches en lactation présentes dans l'exploitation et des performances des animaux.

\section{Conduite alimentaire}

L'analyse du tableau V montre que les sous-produits agro-industriels et les éléments minéraux prenaient une part importante dans l'alimentation du bétail. Les prix des intrants zootechniques ont varié en fonction des saisons. Ainsi, en saison sèche froide, les prix étaient plus bas puisque cette saison suivait la période de récolte. C'était d'ailleurs à ce moment que les exploitants en profitaient pour faire leurs stocks.

\section{Stratégies de valorisation de la production \\ En milieu urbain}

Sur l'ensemble de l'échantillon, il y avait deux traites quotidiennes, mais la traite matinale représentait 60 p. 100 de la production journalière d'une vache. Cette production de lait était vendue à l'état frais par la majorité des éleveurs (88 p. 100). Le reste était destiné à l'autoconsommation et aux dons, surtout chez les intra-urbains chez qui on rencontrait «l'élevage de case » (figure 3).

La plus grande partie des ventes à la laiterie portait sur la traite du matin, tandis que la traite du soir était l'objet de vente par les femmes faisant du porte-à-porte. Le prix du litre de lait frais était de 225, 235 et $250 \mathrm{Fcfa}$, respectivement en saison froide, saison des pluies et saison chaude pour les couronnes 1 et 2, et de 180 Fcfa en toute saison pour la couronne 3. Les dépenses pour l'alimentation étaient plus élevées dans les deux premières couronnes, ce qui justifiait la différence de prix avec la troisième. Les recettes occasionnées par la vente du lait étaient plus importantes en saison des pluies et en début de saison froide, du fait de l'abondance de la production laitière dans tous les sites visités.

\section{Tableau V}

Conduite alimentaire au niveau de la commune rurale de Filingué (CRF) et de la communauté urbaine de Niamey (CUN)

\begin{tabular}{lrr} 
Modes de conduite & CRF (\%) & CUN (\%) \\
alimentaire pratiquée & 84 & 100 \\
\hline Pâturage & 31 & $/$ \\
Transhumance & 2 & $/$ \\
Alimentation à l'auge & & \\
Type de complémentation utilisée & & $/$ \\
Tourteau de coton & 47 & 1 \\
Graine de coton & 73 & 62 \\
Sel & 100 & 93 \\
Son de céréales & 96 & 9 \\
Autre type de complément & 16 & \\
& & \\
Aliment grossier distribué à l'auge & & 11 \\
Foin de Bourgou & $/$ & 23 \\
Paille de brousse & $/$ & 63 \\
Natron & $/$ & 58 \\
Bloc à lécher & $/$ & \\
Résidus de récolte & $/$ &
\end{tabular}

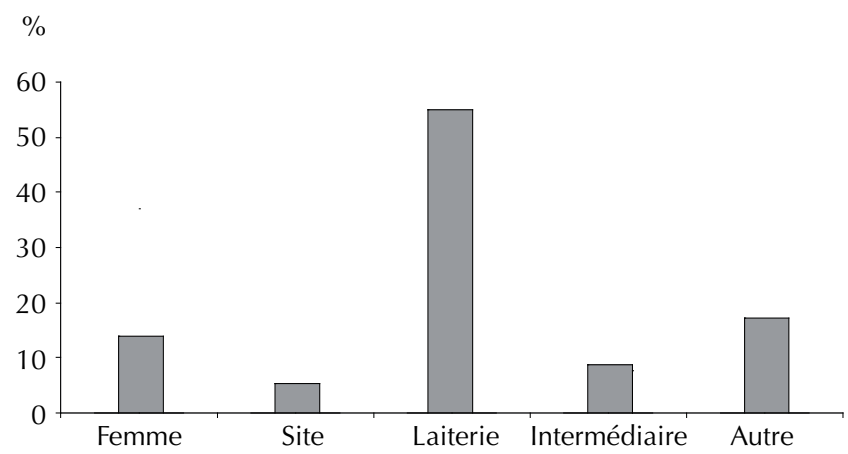

Figure 3 : les différentes modalités de la commercialisation du lait. Femmes : proportion vendue par les femmes dans les marchés ruraux ou urbains; site : proportion vendue au niveau du site ; laiterie : proportion vendue au niveau des laiteries ; intermédiaire : proportion achetée par les revendeurs.

\section{En milieu rural}

La situation était très différente en milieu rural, car les principaux produits laitiers commercialisés étaient le lait caillé (96 p. 100 des enquêtés), le beurre fondu (98 p. 100) et le fromage (10 p. 100). Hormis la transformation en ces produits, le lait frais n'était utilisé que pour l'autoconsommation.

Le lait caillé (ou nono) est obtenu par fermentation du lait frais pendant 24 à 48 heures. Le beurre fondu (ou man chanou ou hawgui) est le produit d'une fermentation poussée (48 à 72 heures), d'un barattage laborieux, suivi d'un rinçage. Enfin, le fromage sec (ou tchoukou) s'obtient par fermentation dirigée par emprésurage à la caillette de veau ou au Camifloc. 
La vente de ces produits laitiers se faisait surtout en priorité par le porte-à-porte (à domicile chez les clients) chez 82 p. 100 des enquêtés, puis au marché (47 p. 100 de l'échantillon), enfin par d'autres voies chez 4 p. 100 d'entre eux.

\section{Les innovations}

Les Peuls, majoritaires dans les deux situations étudiées, sont par tradition des éleveurs et ils maîtrisent par conséquent les pratiques d'élevage. Pour eux, la diversification réside dans l'option laitière au niveau de l'élevage, notamment comment elle peut être un moyen efficace et durable de diversification des revenus et dans quelles conditions. Leur installation à la périphérie des villes leur permet de passer d'une économie pastorale dominée par l'autoconsommation, à une économie de marché tournée vers la vente (6). Les Zarmas et les Haoussas sont plutôt des agriculteurs et l'élevage laitier représente une diversification des sources de revenus.

Dans la CUN, les innovations observées chez les éleveurs enquêtés portaient non seulement sur la conduite du troupeau, mais aussi sur la recherche du meilleur profit. La complémentation de la vache laitière était assez largement répandue et permettait de produire même en saison sèche. Néanmoins, l'insuffisance des quantités distribuées ne permettait pas aux femelles de produire de façon optimale. Par ailleurs, la nécessité de fournir du lait de bonne qualité aux industries laitières a entraîné le développement de la collecte de cette denrée, dont l'efficacité reste à améliorer.

Etant donné l'existence du marché, la possibilité de conserver le lait frais (produit le plus rémunérateur) aussi longtemps que possible a amené les producteurs à le chauffer avant de le destiner à la vente. De même, sous l'impulsion de la FAO, le système lactopéroxydase a également été introduit à Niamey, mais son utilisation n'a pas eu de succès. Des antibiotiques étaient aussi souvent ajoutés dans le lait dans le but de freiner le développement bactérien, avec des conséquences négatives au niveau des industries (fabrication de yaourt impossible) et certainement au niveau de la santé humaine. Le mouillage pouvait aussi être rencontré.

En milieu rural, le marché n'étant pas développé, les initiatives portaient sur la transformation du lait en produits de longue conservation (beurre, fromage...). L'alimentation étant un vrai problème en milieu rural, la production de lait dans ce contexte est pratiquement insuffisante en saison sèche et chaude.

\section{DISCUSSION}

\section{Contraintes des producteurs laitiers}

Le développement de la filière lait autour de Niamey est assez récent et, comme pour les autres grandes villes africaines, il répond à une augmentation de la demande urbaine, elle-même consécutive à l'accroissement de la population et à l'augmentation du niveau de vie (12). La structure du bassin laitier de Niamey reflète l'évolution du contexte qui est caractérisé par une juxtaposition de catégories d'éleveurs dont les objectifs sont le plus souvent différents. Pour la CUN, les contraintes portent de façon globale sur la production et la conservation d'un lait frais de bonne qualité jusqu'au transformateur ou au consommateur.

L'examen de la typologie des élevages a fait ressortir une répartition sous forme de couronnes concentriques de la ville vers sa périphérie. Ainsi, la couronne 1 était composée des élevages appartenant pour la plupart à des éleveurs dits du dimanche (commerçants, fonctionnaires...). Ces élevages étaient implantés dans les vieux quartiers de Niamey (Gamkalley, Saga, Kirkissoye, Lamordé...), dans les propriétés des producteurs, généralement sous la garde d'un berger peul salarié. De façon spécifique, il a surtout été relevé le faible niveau d'organisation des acteurs et le faible encadrement technique.

La couronne 2 était composée de nouveaux venus, en majorité de Peuls dont le début de l'installation remontait aux années 1974 et 1984, périodes correspondant aux dernières grandes sécheresses du Sahel. Ces élevages étaient caractérisés par une grande précarité car installés sur des terrains privés ou communautaires. L'objectif de ces élevages était purement économique et les revenus tirés de la vente du lait représentaient le plus souvent la seule source de survie (11). Etant sur des terres appartenant à d'autres personnes, se posait un problème foncier pour ces éleveurs qui ne pouvaient faire un investissement durable dans des domaines comme l'habitat des animaux. Aussi, l'approvisionnement en intrants zoo-vétérinaires faisait défaut. Enfin, la collecte du lait n'était pas organisée car cette couronne s'étendait jusqu'à neuf kilomètres autour du centre urbain, posant le problème d'accès au marché.

La couronne 3 était composée des villages environnant la capitale où les éleveurs pratiquaient un type d'élevage extensif. A l'origine, ces éleveurs ne vendaient pas de lait à Niamey et préféraient écouler leur production dans les marchés ruraux sous forme de lait caillé (11). Actuellement, avec le développement du réseau routier et des industries laitières qui garantissent l'achat à un prix compétitif, ils se tournent de plus en plus vers la ville.

Dans la couronne 3 qui s'étendait de 10 à $30 \mathrm{~km}$ autour de la ville, on retrouvait également le faible niveau d'organisation des producteurs, l'approvisionnement en intrants zoo-vétérinaires déficient et le problème de collecte. A cela s'ajoutait l'abreuvement difficile, surtout en saison sèche.

Pour la CRF, il s'agissait d'un système d'élevage à faible niveau d'utilisation d'intrants et géré selon le mode extensif. La productivité du cheptel était faible et liée aux fortes variations qualitatives et quantitatives des parcours, voire à leur extrême pauvreté, et aux énormes difficultés d'abreuvement en saison sèche. Il y avait ainsi une véritable saisonnalité de la production laitière avec une chute de production en saison sèche.

De façon cruciale, c'est essentiellement le problème de débouchés (par la faiblesse et la non-structuration du marché) qui a été souligné par la presque totalité des producteurs. Etant donné la limitation de la demande en lait frais, ce dernier était surtout transformé (lait caillé, fromage, beurre) pour pouvoir être conservé pour une vente ultérieure, ce qui rapportait moins en terme de revenu (par exemple 11 de lait frais coûtant 180 à $250 \mathrm{Fcfa}$ permettait de faire une feuille de fromage vendue à $125 \mathrm{Fcfa}$, d'où un manque à gagner pouvant aller de 55 à $125 \mathrm{Fcfa})$.

\section{Options de développement}

Dans la CUN, l'amélioration des performances des animaux exige d'organiser l'approvisionnement en intrants zoo-vétérinaires et d'assurer une vulgarisation rapprochée de thèmes techniques et de pratiques innovantes (comme les cultures fourragères), donc de former les producteurs; il y a nécessité de mieux structurer les acteurs afin d'assurer l'encadrement de proximité.

L'organisation de la collecte est fondamentale pour acheminer le lait dans de bonnes conditions vers les industries laitières. Un certain nombre de producteurs (hommes), qui vendaient dans le centre urbain (aux laiteries, particuliers...), livraient eux-mêmes à vélo ou avec d'autres moyens de locomotion et retournaient à l'exploitation après avoir acheté le son dans des moulins ou en faisant du porte-à-porte. Par ailleurs, Vias et coll. (11) ont décrit l'existence de collecteurs qui se sont spécialisés dans la collecte du lait auprès 
des producteurs pour le revendre directement aux consommateurs ou aux unités de transformation. Compte tenu du potentiel laitier existant en zone périurbaine de Niamey, la création d'un centre de collecte permettra de le valoriser et surtout de réduire le temps de livraison ; le fait que les exploitations soient concentrées le long des sept axes routiers est un atout dans ce sens.

La collecte et, en aval, la distribution devraient être des activités telles qu'elles ont été décrites par Bonfoh et coll. au Mali (1). La vulgarisation de moyens de conservation du lait devrait permettre non seulement d'accompagner le développement du réseau de collecte proposé pour la livraison d'un lait de qualité, mais aussi de trouver une issue pour la production du soir qui pose problème à l'heure actuelle. En dehors du délai de livraison, les ustensiles des acteurs (bergers, collecteurs et vendeurs) jouent énormément sur la qualité microbiologique du lait (1). Dans le cas de la présente étude, il importe d'enquêter sur le niveau de contamination du lait en vue de l'améliorer.

La sécurisation de l'élevage périurbain demande également d'avoir une politique foncière adaptée (7), notamment pour les producteurs de la couronne 2, et une politique en hydraulique pastorale pour les éleveurs de la couronne 3 .

En zone rurale, l'insuffisance de points d'eau d'abreuvement est cruciale, notamment en saison sèche ; cela incite à développer une politique d'hydraulique pastorale adaptée. Par ailleurs, ne pouvant agir sur la demande qui est fonction de la population humaine, la production de lait cru serait drainée vers les centres urbains les plus proches. Ainsi, la collecte serait organisée par la création de centres dans cette zone (comme proposé pour la CUN) pour un acheminement vers la CUN qui est capable d'absorber la production de ces zones, comme cela se fait avec la production de la station sahélienne expérimentale de Toukounous qui livre une part importante de sa production à une laiterie de Niamey tous les deux ou trois jours. En accord avec la FAO (4), ces centres se justifient notamment lorsque les zones de ramassage sont éloignés de l'usine ou lorsque celles-ci sont constituées de petits fournisseurs dont l'accès à leur exploitation est difficile pour des raisons diverses (entre autre l'état défectueux des routes). D'une part, ces centres facilitent l'approvisionnement en lait des usines et, d'autre part, ils incitent les producteurs à accroître leur production sachant que celle-ci est assurée d'un débouché.

\section{CONCLUSION}

Les potentialités sont importantes pour le développement de la filière. En milieu périurbain la présence d'usines de transformation constitue un atout pour la valorisation du lait frais. En milieu rural, l'accent doit être mis pour l'instant sur les techniques de transformation artisanales. Dans les deux situations, les principales contraintes sont l'absence de circuits de collecte performants et la faible organisation des acteurs.

Aussi, la réflexion sur les stratégies de développement de l'élevage laitier et en particulier l'élevage périurbain, dont les objectifs ont été clairement affichés par les pouvoirs publics, doit tenir compte de certaines réalités, notamment la disponibilité alimentaire, les conditions techniques de production et le pouvoir d'achat de la population.

\section{BIBLIOGRAPHIE}

1. BONFOH B., FANE A., NETOYO L., MBAYE Y., SIMBE C.F., ALFAROUK I.O., NICOLET I., FARAH Z., ZINSSTAG I., 2003. Collecte et distribution du lait produit localement en zone urbaine de Bamako. Etud. Rech. sahéliennes (8-9) : 13-18.

2. CANTAFORA A.F.A., 2002. Lo sviluppo della filiera latte nella cintura periurbana di Niamey (Niger). Laurea in Scienze e Technologie della Produzione animale, Intituto di Zootecnica, Universita' Degli Studi, Milano, Italia, $107 \mathrm{p}$.

3. DIRECTION DE LA STATISTIQUE ET DES COMPTES NATIONAUX, 2003. Recensement général de la population et de I'habitat. Niamey, Niger, direction de la Statistique et des Comptes nationaux.

4. FAO, 1985. Réfrigération du lait à la ferme et organisation des transports. Rome, Italie, FAO, 215 p. (Production et santé animales)

5. METZGER R., CENTRES J.M., THOMAS L., LAMBERT J.C., 1995. L'approvisionnement des villes africaines en lait et produits laitiers, un potentiel pour le développement rural. Rome, Italie, FAO, 102 p. (Production et santé animales)

6. MEYER C., DENIS J.P., 1999. Elevage de la vache laitière en zone tropicale. Montpellier, France, Cirad-emvt, 314 p. (Techniques)

7. MINISTERE DES RESSOURCES ANIMALES, 2001. Etat des lieux, axes d'intervention et programmes prioritaires. Document cadre pour la relance du secteur de l'élevage. Niamey, Niger, ministère des Ressources animales, $107 \mathrm{p}$.

8. MOUNKAILA M., 2005. Caractérisation préliminaire des élevages laitiers périurbains de la communauté urbaine de Niamey. Mémoire Ingénieur Techniques agricoles, faculté d'Agronomie, université de Niamey, Niger, 64 p.

9. STAAL S., 1995. Peri-urban dairying and public policy in Ethiopia and Kenya. PhD Dissert., University of Florida, Gainesville, FL, USA, 255 p.

10. THEBAUD B., 1988. Elevage et développement au Niger. Quel avenir pour les éleveurs du Sahel ? Genève, Suisse, BIT, 138 p.

11. VIAS FRANCK S.G., BONFOH B., DIARRA A., NAFERI A., FAYE B., 2003. Les élevages laitiers bovins autour de la communauté urbaine de Niamey : caractéristiques, productions, commercialisation et qualité du lait. Etud. Rech. sahéliennes (8-9) : 159-165.

12. VIAS FRANCK S.G., MARICHATOU H., KORE H., 2005. Synthèse sur les filières laitières au Niger. Atelier de lancement sur " politiques laitières ». Dakar, Sénégal, Eismv, 45 p.

Reçu le 16.05.2007, accepté le 31.10.2007 


\section{Summary}

Boukary A.R., Chaïbou M., Marichatou H., Vias G. Characterization of Dairy Production Systems and Analysis of Milk Promotion Strategies in Rural and Urban Areas in Niger: Case of the Urban Community of Niamey and Rural District of Filingue

Livestock breeding and particularly milk production play a major role in poverty alleviation and economic growth. The present study aimed at characterizing the production systems and opening avenues for milk production in a (sub)urban [urban community of Niamey (UCN)] and in a rural environment [rural district of Filingue (RDF)] in Niger. In UCN, surveys were carried out in 35 dairy sites randomly selected among the 150 already indexed within a radius of $50 \mathrm{~km}$ from the capital. Out of these, 12 sites were selected allowing the questionnaire to be administered to 169 heads of household. In RDF, 49 heads of household, located in five villages within $75 \mathrm{~km}$ of Filingue, were surveyed. Results showed that in UCN, breeders owned few dairy cows (five on average, i.e. $28 \%$ of the bovine herd), which produced in all seasons 7 to $10 \mathrm{~L} /$ household/day; they marketed fresh milk more often than in RDF because they had access to dairy transformation units. In RDF, they owned more cows (ten on average, i.e. $52 \%$ of the bovine herd), which produced only during the rainy season and the cold dry season (between 0 to 10 and 10 to $20 \mathrm{~L} / \mathrm{hou}$ sehold/day according to 66 and $20 \%$ of the persons surveyed, respectively); dairy products were transformed more often before sale (melted butter, curdled milk, cheese). The innovations observed in the surveyed breeders were related to changes in herd management. The constraints to dairy production development in the urban area concerned in particular production and preservation of good-quality fresh milk all the way to transforming units or consumers, while in the rural area, it concerned the lack of avenues. In urban areas, it is essential to organize the supply of food inputs, evening collection of milk and to popularize technical topics and innovating practices.

Keywords: Dairy cattle - Milk production - Milk products Rural area - Suburban area - Niger.

\section{Resumen}

Boukary A.R., Chaïbou M., Marichatou H., Vias G. Caracterización de los sistemas de producción lechera y análisis de las estrategias de valorización de la leche en medio rural y peri urbano en Níger: caso de la comunidad urbana de Niamey y de la comuna rural de Filingué

La cría y particularmente la producción de leche ocupan un lugar preponderante como factor de reducción de la pobreza y del crecimiento económico. El presente estudio tiene como objetivo el de caracterizar los sistemas de producción y los procesos de diversificación hacia la leche en medio peri urbano [comunidad urbana de Niamey (CUN)] y en medio rural (comuna rural de Filingé (CRF)) en Níger. Se realizaron encuestas en la CUN en 35 sitios lecheros escogidos de manera aleatoria entre 150 registrados anteriormente en un radio de $50 \mathrm{~km}$ alrededor de la capital. Una escogencia cuidadosa de 12 sitios permitió la administración de un cuestionario a 169 cabezas de hogar. En la CRF, se entrevistaron 49 cabezas de hogar, repartidos en cinco pueblos, situados en un radio de $75 \mathrm{~km}$ alrededor de Filingué. Los resultados muestran que en la CUN los criadores poseían un pequeño número de vacas lecheras (cinco vacas en promedio, sea $28 \%$ del hato bovino); la producción se extendía durante todas las estaciones y era de 7 a 10 l/hogar/día; a menudo la leche fresca era comercializada únicamente en CRF debido a la presencia de unidades de transformación de leche. En la CRF, por el contrario, los efectivos eran más importantes (un promedio de 10 vacas lecheras, sea $52 \%$ del hato bovino); las hembras sólo producían durante la época de lluvias y la seca fría (0 a $10 \mathrm{l} /$ hogar/día para $66 \%$ de los entrevistados y entre 10 y 20 I para $20 \%$ de los entrevistados): los productos lecheros eran a menudo transformados antes de ser vendidos (mantequilla derretida, leche cortada, queso). Las innovaciones observadas en los criadores interrogados se refirieron a las transformaciones y las modificaciones en la conducta del hato. Los obstáculos al desarrollo de la producción de leche provenían, en medio urbano, al problema de la producción y la conservación de la leche fresca de buena calidad hasta el transformador o consumidor y en medio rural, del problema de la demanda del producto. Es necesario, en medio urbano, organizar el abastecimiento de productos alimenticios, la colecta de la leche en la noche y proceder a una divulgación de cercanía de los temas técnicos y de las prácticas innovadoras.

Palabras clave: Ganado de leche - Producción lechera Producto lácteo - Zona rural - Zona periurbana - Níger. 